\title{
Surgery for Patients Undergoing Anticoagulant Therapy
}

\author{
Karpaga Preethitha .S
}

\begin{abstract}
Dental surgeries in patients under anticoagulant therapy are considered crucial and are done after careful monitoring of the patient's medical history and blood reports. This is due to the uncontrolled bleeding that is possible to occur following invasive treatment in such patients.Perioperative management of chronically anticoagulated patients and/or patients treated with antiplatelet therapy is a complex medical problem (1).More than half of all hematologists, oral surgeons, and maxillofacial surgeons surveyed in Ontario in 2005 routinely interrupted anticoagulation therapy for tooth extractions (2). Surveys from other countries report that many dental professionals are unaware of their patients' coagulation status, and still discontinue antiplatelet therapy unnecessarily (3). Thus In 1954, the American Heart Association recommended a therapeutic range for oral anticoagulant therapy of a prothrombin time ratio (PTR) of $2-2.5$ using human brain reagents (4). This article deals with the various methods available to avoid the uncontrolled bleeding following dental treatments in anticoagulated patients.
\end{abstract}

Keywoords: anticoagulants, antiplatelet drugs, preoperative management

\section{Introduction}

Anticoagulants are a type of drug that reduces the body's ability to form clots in the blood (5). These drugs act by inhibiting one or more clotting factors that are essential for the clot formation. Although they are sometimes called blood thinners, they do not actually thin the blood. This type of medicine will not dissolve clots that already have formed, although it will help to stop an existing clot from getting larger (5).

Anticoagulant drugs are commonly prescribed for patients who have undergone cardiac surgeries or patients under the risk of ischemic stroke, myocardial infraction, atrial fibrillation, pulmonary embolism, deep vein thrombosis, venous thromboembolism, congestive heart failure, stroke, myocardial infarction, and genetic or acquired hypercoagulability. A side effect of all anticoagulants is the risk of excessive bleeding (haemorrhages). This is because these medicines increase the time it takes blood clots to form (6).

The gums and their supporting structures are highly vascular and prone to bleeding when damaged (7). While visible bleeding in the mouth is not considered as dangerous as "silent" bleeding that occurs internally or at noncompressible sites, oral bleeding can be distressing for the patient and potentially life threatening (8). Invasive procedures such as local infiltration, scaling below the gums, root planning, biopsies, tooth extractions, minor periodontal surgery, cavity filling, endodontic procedures (root canals), and prosthodontic procedures (crowns, bridges, and implants) are also unlikely to cause significant bleeding. Significiant bleeding is more likely to occur with more invasive procedures such as extraction of impacted teeth and use of periodontal flaps (9) (10)(11)(12)

Hence a proper knowledge on the on the type of drug the patient consumes, duration, dosage of the drug and the estimation of blood for the level of clotting factors are necessary to reduce the risk and effective management of such patients.
Commonly used anticoagulants and their mechanism of action:

\section{Heparin:}

Heparin, used primarily in hospitalized patients, is a mixture of mucopolysaccharides that promote the activity of antithrombin III, a blood plasma protein that inactivates thrombin (an enzyme that promotes clotting). Because it is not well absorbed from the gastrointestinal tract, heparin is given intravenously to inhibit coagulation immediately, or it is given subcutaneously. The major side effect associated with heparin is hemorrhage; thrombocytopenia (reduced number of circulating platelets) and hypersensitivity reactions also may occur. When oral anticoagulants are given with heparin, additional anticoagulant effects occur (13).

\section{Coumarin:}

The coumarin derivatives resemble vitamin $\mathrm{K}$, an important element in the synthesis of a number of clotting factors. Interference in the metabolism of vitamin $\mathrm{K}$ in the liver by coumarin derivatives gives rise to clotting factors that are defective and incapable of binding calcium ions (another important element in the activation of coagulation factors at several steps in the coagulation cascade). Warfarin, a coumarin derivative and the most commonly used oral anticoagulant, is rapidly and almost completely absorbed orally.Haemorrhage is the principal toxic effect during oral anticoagulant therapy (13)

\section{Anti-platelet drugs:}

- Platelets provide the initial haemostatic plug at the site of a vascular injury. They are also involved in pathological processes and are an important contributor to arterial thrombosis leading to myocardial infarction and ischaemic stroke.

Available antiplatelet medications include:

- Low-dose aspirin (75mg-300mg daily). Used for the secondary prevention of thrombotic1 cardiovascular or cerebrovascular disease and following coronary artery bypass surgery.

- Clopidogrel (Plavix $®)$. Licensed as monotherapy for the prevention of atherothrombotic events in patients 


\section{International Journal of Science and Research (IJSR) \\ ISSN (Online): 2319-7064}

Index Copernicus Value (2015): 78.96 | Impact Factor (2015): 6.391

suffering myocardial infarction, ischaemic stroke or peripheral arterial disease and in conjunction with aspirin for non-ST segment elevation acute coronary syndrome (unstable angina or non-Q-wave myocardial infarction) and ST segment elevation acute myocardial infarction in medically treated patients eligible for thrombolytic therapy.( 14) Clopidogrel is also recommended in combination with low-dose aspirin after the insertion of a coronary artery stent (unlicensed indication).

- Dipyridamole (Persantin®, Persantin Retard®). Used as an adjunct to oral anticoagulation for the prophylaxis of thromboembolism associated with prosthetic heart valves. (15)

All antiplatelet medications affect clotting by inhibiting platelet aggregation but they do so by a variety of different mechanisms. Aspirin irreversibly acetylates cyclooxygenase, inhibiting the production of thromboxane A2. This results in decreased platelet aggregation by adenosine diphosphate (ADP) and collagen. Clopidogrel selectively inhibits ADPinduced platelet aggregation. Dipyridamole is an adenosine reuptake inhibitor and phosphodiesterase inhibitor with antiplatelet and vasodilating activity. 6 The action of dipyridamole is reversible. Aspirin begins irreversibly inhibiting platelet aggregation within one hour of ingestion and clopidogrel within two hours; this lasts for the life of the platelets (7-10 days). The effect is only overcome by the manufacture of new platelets. 8 Complete recovery of platelet aggregation may occur in $50 \%$ of cases by day three and in $80 \%$ of cases by day four.(16)

Tests to asses the patients fitness prior dental surgery:

INR values before treatment:

It stands for International Normalised Ratio (INR) and is a measure of how much longer it takes the blood to clot when oral anticoagulation is used

In patients receiving long-term anticoagulant therapy and who are stably anticoagulated on warfarin an INR check 72 hours prior to surgery is recommended. This allows sufficient time for dose modification if necessary to ensure a safe INR (2-4) on the day of dental surgery.

There is no need to check the INR for non-invasive dental procedures although scaling subgingivally will require an INR check.

The INR should also be checked if performing an inferior alveolar nerve block (IANB) as there is an anecdotal risk of haematoma and airway compromise. If needed, an IANB should be given cautiously, using an aspirating syringe, with an INR $<3.0$.

Prothrombin time:

Patients who use OAC( oral anti-coagulant) have their therapy monitored by measuring the Prothrombin Time (PT). This test measures the time for clot formation from VII factor activation to fibrin coagulum formation. Due to variations in the methodology, reagents, and instruments used in each laboratory, a normalization ratio was established for PT measurements (INR)
Normal Values and Critical Limits: 8.8 - 11.6 seconds.

Heparin at therapeutic doses usually does not interfere with the PT, but PT prolongation can result in patients receiving higher doses of Heparin. In fact, due to variability in the sensitivities of different thromboplastins to heparin, a falsely prolonged PT can occur during initiation of warfarin treatment when the patient is simultaneously receiving heparin therapy.

Dental treatment and anticoagulant drug management:

\section{Local measures:}

Tranexamic acid binds to plasminogen and inhibits subsequent lysis of fibrin. Orally administered tranexamic acid does not appear in saliva at detectable levels (16) but as a mouthwash, the concentration of tranexamic acid remains sufficiently high to inhibit fibrinolysis for hours but insignificant levels are detected in the plasma.

The use of gelatin sponges and sutures provided adequate haemostasis for dental extraction without interruption of anticoagulants. Tranexamic acid mouthwashes or fibrin glue offered no benefit over resorbablegelatin sponges plus suturing. In over $99 \%$ of cases the use of resorbable gelatine sponges plus sutures at the time of dental surgery was sufficient to achieve haemostasis and only 1 case of severe bleeding was observed. However, all patients received tranexamic acid mouthwashes every 6 hours for 2 days. In all groups receiving oral anticoagulants, local haemostasis was secured with oxidised cellulose and silk sutures plus local application of a tranexamic acid saturated gauze swab for 30-60 minutes following surgery. There was no difference in bleeding complications.(17)

Other local methods:

- local pressure (biting on gauze or tea bags)

- electrocautery

- topical thrombin

- mouth rinse(s)

* cold water o aminocaproic acid 5\% mouth rinse (5 grams in $100 \mathrm{ml}$ of sterile water)

* Note: this solution may be difficult to obtain from a pharmacy unless prior arrangement have been made

* hold $10 \mathrm{ml}$ in mouth for 2 min $1 / 2$ hour pre-procedure then repeat $\mathrm{q} 2 \mathrm{~h}$ for 6-10 doses prn

- avoid additional bleeding risks (hot liquids, other mouth washes, and hard foods) for at least $24 \mathrm{hrs}$

Stoppage of drugs before dental procedure:

\begin{tabular}{|l|c|c|}
\hline Dental procedure & $\begin{array}{c}\text { Presumed } \\
\text { Bleeding } \\
\text { Risk }\end{array}$ & $\begin{array}{c}\text { Peri-procedural } \\
\text { recom- } \\
\text { mendations* }\end{array}$ \\
\hline $\begin{array}{l}\text { Supraginival scaling } \\
\text { Simple restorations } \\
\text {. Local anesthetic injections }\end{array}$ & low & $\begin{array}{c}\text { Continue } \\
\text { therapeutic } \\
\text { anticoagulation }\end{array}$ \\
\hline $\begin{array}{l}\text { Subginival scaling } \\
\text { Subgingival preparation restoration } \\
\text { Standard root canal } \\
\text { Simple extractions } \\
\text { Regional anesthetic injections }\end{array}$ & medium & $\begin{array}{c}\text { Continue } \\
\text { therapeutic } \\
\text { anticoagulation }\end{array}$ \\
\hline $\begin{array}{l}\text { Extensive surgery } \\
\text { Apicoectomy (root removal) } \\
\text { Alevolar surgery (bone removal) }\end{array}$ & high & $\begin{array}{c}\text { Consider } \\
\text { reducing } \\
\text { anticoagulation }\end{array}$ \\
\hline
\end{tabular}




\section{International Journal of Science and Research (IJSR) \\ ISSN (Online): 2319-7064}

Index Copernicus Value (2015): 78.96 | Impact Factor (2015): 6.391

When extensive surgery is necessary and it is has been determined to lower the level of anticoagulation, the following can be considered as a guide in the pre-procedural period:

\section{Warfarin}

- Withholding warfarin 2 to 7 days prior to procedure depending on the indication for and goal of anticoagulation. Longer holds should be considered for patients with either advanced age, systolic heart failure, or requiring low $(<3 \mathrm{mg} /$ day) dosing.

- Possibly initiate a parenteral anticoagulant peri-procedure [heparin or low molecular weight heparin (LMWH)]

- Warfarin and the parenteral anticoagulant (if necessary) should be restarted when deemed appropriate and safe after the procedure, and the parenteral anticoagulant can be discontinued when the INR is within therapeutic range

\section{Apixaban}

- Hold apixaban 1-2 days prior to procedure if $\mathrm{CrCL}>50$ $\mathrm{mL} / \mathrm{min}$

- Hold apixaban 1-4 days prior to procedure if $\mathrm{CrCl} 30$ - 50 $\mathrm{mL} / \mathrm{min} \bullet$ Hold apixaban 2-4 days prior to procedure if $\mathrm{CrCl}<30 \mathrm{~mL} / \mathrm{min}$

- Restart apixaban when deemed appropriate and safe after procedure .

The ACCP 2008 guidelines for antithrombotic and thrombolytic therapy recommend In patients who require a minor dental procedure, we suggest continuing VKAs with an oral prohemostatic agent or stopping VKAs (warfarin) 2 to 3 days before the procedure instead of alternative strategies (Grade 2C). (18)(19).

\section{Conclusion}

Dental surgery in anticoagulated patients is common and historically their management has been controversial following early reports of major bleeding in such individuals.(20).Many of the early reports of haemorrhage associated with dental surgery during this period predated the standardisation of oral anticoagulant control by means of the INR. In 1954, the American Heart Association recommended a therapeutic range for oral anticoagulant therapy of a prothrombin time ratio (PTR) of 2-2.5 using human brain reagents (21). Later, the use of less sensitive commercial thromboplastins was not accompanied by a change in the target PTR ratio. Clinicians, therefore, administered larger doses of oral anticoagulants to achieve the target ratio, resulting in an increased incidence of haemorrhage. There is no definite protocol or method that is used for the management of bleeding in antcoagulated patients. The management depends on the nature,dosage,duration of drug and the INR levels of the patient.

\section{References}

[1] Institute for Clinical Pharmacology, Pharmazentrum Frankfurt, University Hospital, Theodor-Stern-Kai 7, D-60590 Frankfurt, Germany. harder@em.unifrankfurt.de
[2] Lim W, Wang M, Crowther M, et al. The management of anticoagulated patients requiring dental extraction: a cross-sectional survey of oral and maxillofacial surgeons and hematologists. J ThrombHaemost. 2007;5:2157-9.)

[3] Murphy J, Twohig E, McWilliams SR. Dentists' approach to patients on anti-platelet agents and warfarin: a survey of practice. J Ir Dent Assoc. 2010;56:28-31)

[4] Wright IS, Beck DF, Marple CD. Myocardial infarction and its treatment with anticoagulants; summary of findings in 1031 cases. Lancet 1954;266(6802):92-5

[5] http://www.anticoagulationeurope.org/conditions/antic oagulation-therapy

[6] http://www.nhs.uk/Conditions/Anticoagulantmedicines/Pages/Side-effects.aspx

[7] Nematullah A, Alabousi A, Blanas N, et al. Dental surgery for patients on anticoagulant therapy with warfarin: a systematic review and meta-analysis. J Can Dent Assoc. 2009; 75: 41a-i. (Available from URL:www.cda-adc.ca/jcda/vol-75/issue-1/41.html

[8] Sandor G. Do patients taking oral anticoagulants need to discontinue their medication before surgical procedures? J Can Dental Assoc. 2004; 70: 482-3.

[9] http://www.dpic.org/article/professional/managementanticoagulant-and-antiplatelet-therapy-patientsundergoing-dental-pr

[10] .Gupta A, et al. Bleeding disorders of importance in dental care and related patient management. Can Dent Assoc J. 2007; 73: 77-83

[11] .Hermann WW, Konzelman JL Jr, Sutley SH. Current perspectives on dental patients receiving anticoaguilant therapy. J Am Dental Assoc. 1997; 128: 327-35

[12] Bacci C, Maglione M, Favero L,et al. Management of dental extraction in patients undergoing anticoagulant treatment. Results from a large, multicentre, prospective, case-control study. ThrombHaemost. 2010;104:972-5.

[13] http://www.britannica.com/science/anticoagulant

[14] Dispersible AspirinTabletsBP 75mg Summary of Product Characteristics, Boots Company PLC (www.emc.medicines.org.uk accessed 22/11/06)

[15] Plavix Summary of Product Characteristics. Sanofi Pharma - Bristol-Myers Squibb SNC, (www.emc.medicines.org.uk accessed 22/11/06).

[16] Persantin/Persantin Retard Summary of Product Characteristics, BoehringerIngelheim Limited (www.emc.medicines.org.uk accessed 22/11/06).

[17] com/documents/WarfarinandentalSurgery_bjh_264_20 07.pdf

[18] http://www.ucdmc.ucdavis.edu/anticoag/pdf/Anticoag DentalProcedure.pdf

[19] Douketis JD et al. CHEST 2012; 141:326-350S.

[20] Ziffer A M, Scopp I W, Beck J, Baum J, Berger A R. Profound bleeding after dental extractions during dicumarol therapy. N Engl J Med 1957; 256: 351-353. | PubMed | ISI | ChemPort

[21] Wright I S, Beck D F, Marple C D. Myocardial infarction and its treatment with anticoagulants; summary of findings in 1031 cases. Lancet 1954; 266: 92-95. PubMed | ChemPort 